\title{
A Modification to the Group Delay and Simulated Annealing Technique for Characterization of Peripheral Nerve Fiber Size Distributions for Non-Deterministic Sampled Data
}

\author{
Robert B. Szlavik
}

\begin{abstract}
The ability to determine the characteristics of peripheral nerve fiber size distributions would provide additional information to clinicians for the diagnosis of specific pathologies of the peripheral nervous system. Investigation of these conditions, using electro-diagnostic techniques, is advantageous in the sense that such techniques tend to be minimally invasive yet provide valuable diagnostic information. One of the principal electro-diagnostic tools available to the clinician is the nerve conduction velocity test. While the peripheral nerve conduction velocity test can provide useful information to the clinician regarding the viability of the nerve under study, it is a single parameter test that yields no detailed information about the characteristics of the functioning nerve fibers within the nerve trunk. In previous work, the efficacy of the group delay and simulated annealing approach was demonstrated in the context of a simulation study where deterministic functions were used to represent the single fiber evoked potentials. In this study we present a modification to the approach discussed previously that is applicable to nondeterministic functions of sampled data.
\end{abstract}

\section{INTRODUCTION}

$\mathrm{T}$ he nerve conduction velocity test provides clinically useful information in the diagnosis of peripheral neuropathies, such as Carpal Tunnel Syndrome [1;2]. Since nerve conduction velocity studies are essentially single parameter measurements of the gross conduction properties of the underlying nerve trunk, such studies are not suited to providing detailed information regarding the characteristics of the underlying nerve fibers that contribute to the compound evoked potential.

A more robust measurement technique would involve the ability to extract information about the viability of the underlying nerve fibers which could potentially provide useful information to the clinician. As an example, information related to the size distribution of contributing nerve fibers can be used to differentiate between different clinical conditions such as Chronic Inflammatory Demyelinating Polyneuropathy, which selectively impacts larger nerve fibers, or Early Diabetic Peripheral Neuropathy, which impacts smaller fibers [3;4].

Manuscript received April 7, 2009

This work was supported in part by the Department of the Navy, Office of Naval Research.

R. B. Szlavik is with the Department of Biomedical \& General Engineering, California Polytechnic State University, San Luis Obispo, CA 93407-0350 USA (phone: 805-756-2025; fax: 805-756-6424; e-mail: rszlavik@calpoly.edu).
There is a large body of literature devoted to describing various techniques for determining the nerve fiber conduction velocity distribution (CVD). The pioneering work of Cummins and Dorfman $[5 ; 6]$ describe techniques that use two compound action potentials to estimate the conduction velocity distribution using a least squares approach. Common to these studies was the assumption that fibers included in a specific velocity class have identical evoked potential waveforms.

More recently there have been several additional studies including the work of Gonzalez-Cueto, Papadopoulou and $\mathrm{Gu}$ [7-9]. The study presented by $\mathrm{Tu}$ et. al. focused on a regularized least squares algorithm but features many of the same assumptions associated with waveform commonality related to velocity classes that were made in earlier work [10]. This study also investigated the impact of noise on the integrity of the estimated CVD.

In several recent publications, we have demonstrated the utility of a group delay based approach for determining the size or conduction velocity distribution of fibers in a peripheral nerve trunk [11]. In a subsequent study, we demonstrated that simulated annealing could be used to optimize the group delay estimate of the nerve fiber diameter distribution in the context of the maximal compound evoked potential template [12]. Both these studies involved simulations where deterministic functions were used to simulate the single fiber evoked potentials. While this approach was useful in demonstrating the overall efficacy of the technique, the method by which the technique would have to be modified to accommodate non-deterministic sampled data was not described and is consequently the focus of this study.

\section{METHOD}

The principal difficulty associated with applying the group delay and simulated annealing approach to nondeterministic sampled data is based on the lack of a suitable time shift, delay reference or temporal marker $\delta_{j}$ that is inherent to the deterministic function used to describe the single fiber action potentials [13]. What is required is the determination of a suitable temporal marker for a nondeterministic function characteristic of what could be expected from the sampled data of the single fiber action potential waveforms $\Gamma^{(1)}(t)$ and $\Gamma^{(2)}(t)$ as per the notation used in previously published work $[11 ; 12]$.

The availability of a suitable time reference for the nondeterministic case is necessary to implement the simulated annealing algorithm utilized to optimize the nerve fiber size 


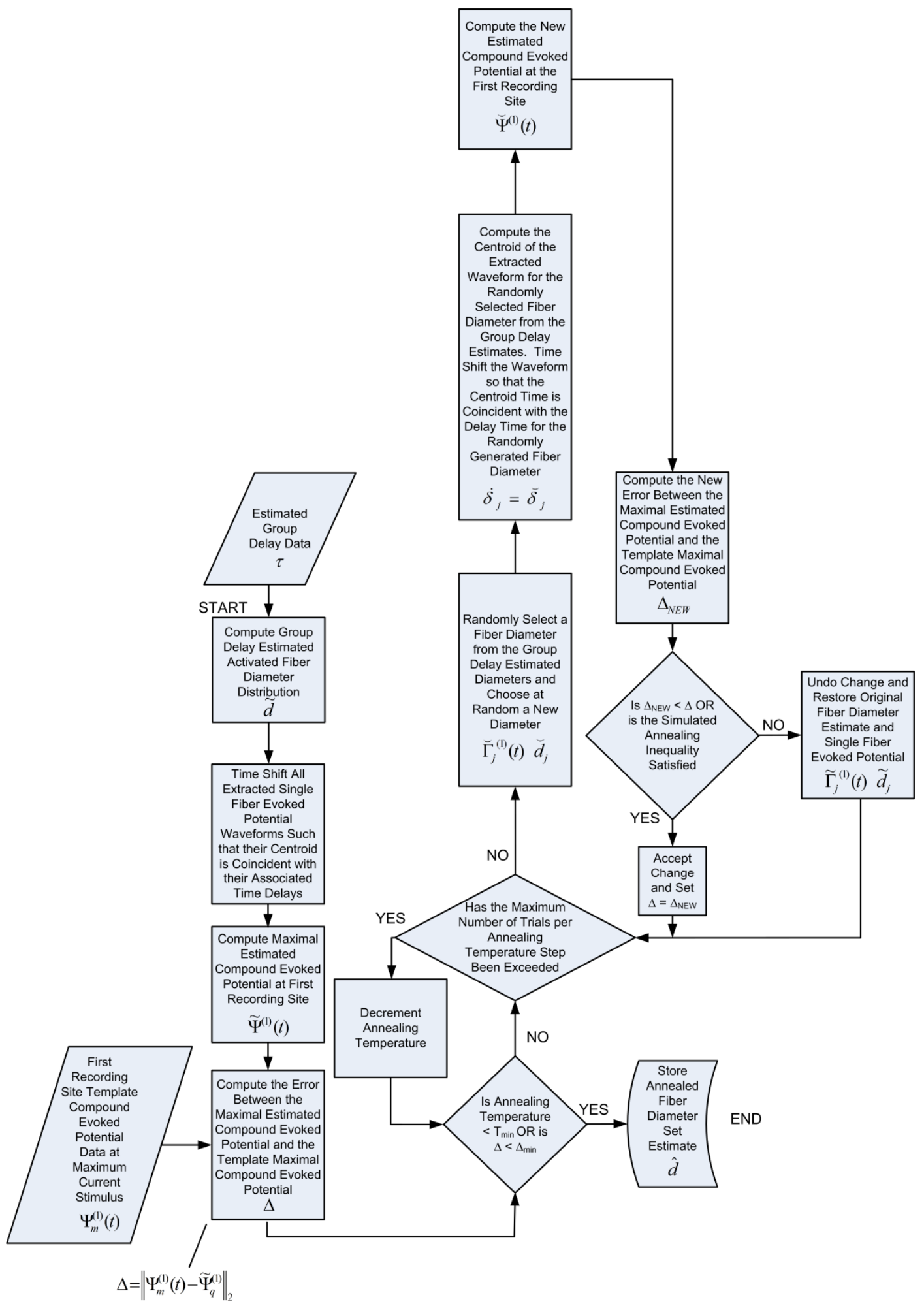

Figure 1. Flowchart of the simulated annealing algorithm that computes an improved estimate of the nerve fiber diameter population set $\widehat{\boldsymbol{d}}$ from the group delay estimated population set $\widetilde{\boldsymbol{d}}$ for non-deterministic sampled data. The flowchart assumes utilization of the first recording site template maximal compound evoked potential although either the first or second site could be used. 
distribution estimate against the maximal compound evoked potential template. This necessity is based on the fact that the simulated annealing approach varies the fiber diameter, and consequently the time delay of the fiber evoked potential, for a randomly chosen fiber in the nerve trunk population.

A physically relevant temporal parameter associated with the individual decomposed single fiber action potential waveforms $\Gamma_{j}^{(n)}(t)$ is the centroid of the absolute value of the waveform in question which may be computed as per (1).

$$
\dot{\delta}_{j}=\frac{\int_{-\infty}^{\infty}\left|t \Gamma_{\mathrm{j}}^{(\mathrm{n})}(\mathrm{t})\right| d t}{\int_{-\infty}^{\infty}\left|\Gamma_{\mathrm{j}}^{(\mathrm{n})}(\mathrm{t})\right| d t}
$$

Since the standard physical interpretation of the centroid is in the context of masses, the functions evaluated in the centroid expression must be positive for all $t$.

Initially, sampled waveforms are shifted such that their centroids are coincident with the propagation delay parameter for each fiber estimate. The centroid parameter for a given single fiber evoked potential waveform is then utilized in the simulated annealing algorithm at the point where a randomly selected fiber $\tilde{d}_{j}$ is chosen from the group delay estimated set $\widetilde{\boldsymbol{d}}$. A new trial fiber diameter $\breve{d}_{j}$ is generated and the original randomly selected fiber diameter is set equal to the new value such that $\tilde{d}_{j}=\check{d}_{j}$. The concomitant delay time $\check{\delta}_{j}$ is computed for the randomly generated fiber diameter. Utilizing the centroid of the associated sampled waveform $\tilde{\Gamma}_{j}^{(n)}(t)$ for the fiber $\tilde{d}_{j}$ as the temporal reference, the time sampled evoked potential waveform $\tilde{\Gamma}_{j}^{(n)}(t)$ is time shifted such that its centroid $\dot{\delta}_{j}$ is aligned with the time delay $\check{\delta}_{j}$. The rest of the simulated annealing algorithm proceeds as described in [12]. Due to the overall complexity of the algorithm, a further description of the modification is given in the flow chart of Figure 1.

\section{RESULTS}

A population of 100 randomly generated fibers was utilized in these studies in accordance with the formula for the randomly generated distribution outlined in previously published work $[11 ; 12]$. Parameters for the specific distribution utilized are given in the caption to Figure 3. These fibers were subjected to a virtual stimulus pulse train of successively increasing amplitudes ranging from zero to a maximum of $1 \mathrm{~mA}$ in $500 \mathrm{nA}$ steps. At each step the compound evoked potential at both virtual recording sites was computed and subsequently, the estimate of single fiber action potential waveforms were obtained at each recording site. The concomitant group delays between the two virtual recording sites were computed yielding the group delay estimated set of fiber diameters $\widetilde{\boldsymbol{d}}$.

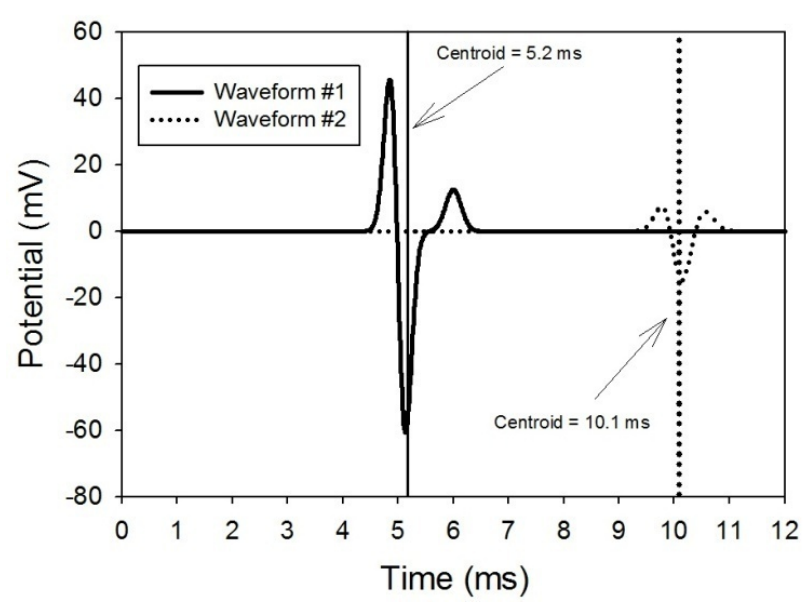

Figure 2. Plot of two typical single fiber evoked potential waveforms showing their centroids, as per (1), computed from a modified form $[11 ; 12]$ of the function proposed by Fleisher [13]. The relevant parameters for Waveform \#1 were $a=5 \mu \mathrm{m}, r=0.5 \mathrm{~mm}, s=1 \cdot a, \sigma_{e}=1 \mathrm{~S} / \mathrm{m}, \alpha=0.998$ and a recording distance of $25 \mathrm{~mm}$. For Waveform \#2, the parameters were $a=5 \mu \mathrm{m}, r=0.5 \mathrm{~mm}, s=0.5 \cdot a, \sigma_{e}=1 \mathrm{~S} / \mathrm{m}, \alpha=0.998$ and a recording distance of $50 \mathrm{~mm}$. In both cases $c=5.0 \times 10^{5} \mathrm{~s}^{-1}$. The parameter values are specified using labels consistent with previously published work $[11 ; 12]$.

The modified simulated annealing technique with the centroid approach described above was then applied to each of the extracted evoked potential waveforms in the set $\boldsymbol{F}^{(2)}(t)$. Results of the centroid computation are shown in Figure 2 for two single fiber evoked potential waveforms with parameters as indicated in the figure caption.

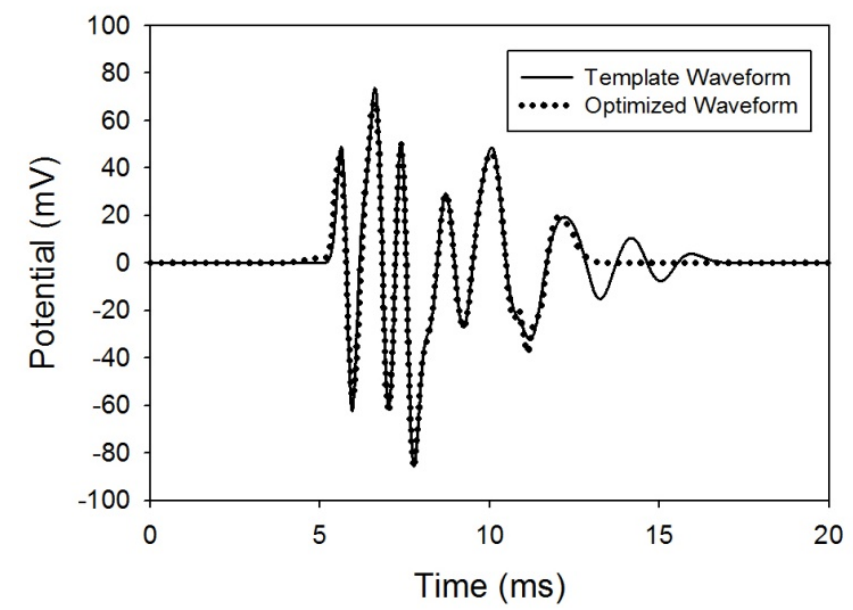

Figure 3. Graph of a maximal compound evoked potential template waveform and the maximal compound evoked potential estimate waveform obtained through group delay estimation and simulated annealing optimization. The simulated annealing algorithm utilized the centroid technique outlined in this paper with the concomitant time shifting of the estimates of the single fiber evoked potential waveforms in $\Gamma^{(2)}(t)$. The relevant simulation parameters for the fiber size distribution were $\beta_{1}=0.35$ $\mathrm{m}, \sigma_{1}=1.699 \mu \mathrm{m}, \mu_{1}=7.5 \mu \mathrm{m}, \beta_{2}=0.65 \mathrm{~m}, \sigma_{2}=1.699 \mu \mathrm{m}$ and $\mu_{2}=13$ $\mu \mathrm{m}$. For simulation of the initial single fiber evoked potentials, the relevant parameters were $r=1 \mathrm{~mm}, c=5 \times 10^{5} \mathrm{~s}^{-1}, s=1 \cdot a, \sigma_{e}=1 \mathrm{~S} / \mathrm{m}, \alpha=0.998, \zeta$ $=10 \mathrm{~mA}$ and $\eta=3.5 \times 10^{5} \mathrm{~m}^{-1}$. The simulated annealing algorithm was implemented with the parameters $T=10, T_{M I N}=1.0 \times 10^{-5}, \Delta_{M I N}=1.0 \times 10^{-4}$ and an annealing step factor of 0.9. The maximum number of trials for each annealing temperature step was limited to 1000 . 
The graph of Figure 3 shows the results of an optimization of a set of estimated singled fiber evoked potentials waveforms at the second recording site $\Gamma^{(2)}(t)$ against the template maximal compound evoked potential waveform. Relevant parameters for this simulation are given in the figure caption.

The chi-square test comparing the actual distribution to the group delay estimated distribution yields $Q\left(\chi^{2} \mid x\right)=$ 0.4697 while the comparison of the actual to the annealed distribution yields $Q\left(\chi^{2} \mid x\right)=0.7000$.

\section{DISCUSSION}

It has previously been demonstrated by the author that the simulated annealing approach can make significant improvements in fiber diameter distribution estimates made using the group delay technique. Based on the modification proposed in this paper, the technique is also beneficial when applied to the extracted estimates of the single fiber evoked potential waveforms where there is no closed form functional description.

A quantitative description of the relevant distributions using the chi-square test is also revealing as to the efficacy of the modified technique. The chi-square test indicates that there is a demonstrable improvement in the fidelity of the fiber diameter distribution obtained after annealing over that of the fidelity of the distribution that results from just the group delay estimation process when compared with the actual distribution.

In the author's experience, it is advantageous to apply the simulated annealing algorithm to data sets at the more distal recording site from the stimulus site. Optimization results at the more distal site yield an improved estimate of the fiber diameter distribution compared to performing the optimization with the more proximal recording site data set.

\section{REFERENCES}

[1] P. A. Parker and P. Kelly, "Nerve conduction velocity measurement techniques," Journal of Clinical Engineering, vol. 7, no. 2, pp. 153158, Apr.1982.

[2] I. Atroshi, C. Gummesson, R. Johnsson, and E. Ornstein, "Diagnostic properties of nerve conduction tests in population-based carpal tunnel syndrome," BMC Musculoskeletal Disorders, vol. 4, no. 9 May2003.

[3] Y. Harati, "Diabetic peripheral neuropathies," Annals of Internal Medicine, vol. 107, pp. 546-559, 1987.

[4] L. J. Dorfman, K. L. Cummins, G. M. Reaven, J. Ceranski, M. S. Greenfield, and L. Doberne, "Studies of diabetic polyneuropathy using conduction velocity distribution (DCV) analysis," Neurology, vol. 33, no. 6, pp. 773-779, June1983.

[5] K. L. Cummins, L. J. Dorfman, and D. H. Perkel, "Nerve fiber conduction-velocity distributions, II Estimation based on two compound action potentials," Electro-Encephal. Clin. Neurophysiol, vol. 46, no. 6, pp. 647-658, 1979.

[6] L. J. Dorfman, "The distribution of conduction velocities (DCV) in peripheral nerves: a review," Muscle \& Nerve, vol. 7, no. 1, pp. 2$11,1984$.

[7] J. A. Gonzalez-Cueto and P. A. Parker, "Deconvolution estimation of nerve conduction velocity distribution," IEEE Transactions on Biomedical Engineering, vol. 49, no. 2, pp. 140-151, Feb.2002.

[8] F. A. Papadopoulou and S. M. Panas, "Estimation of the nerve conduction velocity distribution by peeling sampled compound action potentials," IEEE Transactions on Magnetics, vol. 35, no. 3, pp. 1801-1804, May1999.

[9] D. Gu, R. E. Gander, and E. C. Crichlow, "Determination of nerve conduction velocity distribution from sampled compound action potential signals," IEEE Transactions on Biomedical Engineering, vol. 43, no. 8, pp. 829-838, Aug. 1996.

[10] Y. X. Tu, A. Wernsdorfer, S. Honda, and Y. Tomita, "Estimation of Conduction velocity distribution by regularized-least-squares method," IEEE Transactions on Biomedical Engineering, vol. 44, no. 11, pp. 1102-1106, Nov.1997.

[11] R. B. Szlavik, "A novel method for characterization of peripheral nerve fiber size distributions by group delay," IEEE Trans. Biomed. Eng, vol. 55, no. 12, pp. 2836-2840, Dec.2008.

[12] R. B. Szlavik and G. E. Turner, "A novel method for characterization of peripheral nerve fiber size distributions by group delay measurements and simulated annealing optimization," in 30th Annual International Conference of the IEEE Engineering in Medicine and Biology Society 2008, pp. 5008-5014.

[13] S. M. Fleisher, M. Studer, and G. S. Moschytz, "MathematicalModel of the Single-Fiber Action-Potential," Medical \& Biological Engineering \& Computing, vol. 22, no. 5, pp. 433-439, 1984. 